\title{
Stability of Viscoelastic Wave Equation with Structural $\delta$-Evolution in $\mathbb{R}^{n}$
}

\author{
A. Beniani ${ }^{1}, \mathrm{Kh}$. Zennir $^{2, *}$ and A. Benaissa ${ }^{3}$ \\ ${ }^{1}$ Center University of Belhadj Bouchaib-B.P. 284 RP, Ain Temouchent 46000, \\ Algeria \\ 2 Department of Mathematics, College of Sciences and Arts, Qassim University, \\ Ar-Rass, Saudi Arabia \\ ${ }^{3}$ Laboratory ACEDP, University of Djillali Liabes-B. P. 89, Sidi Bel Abbes 22000, \\ Algeria
}

Received 27 October 2017; Accepted (in revised version) 15 May 2018

\begin{abstract}
The aim of this paper is to study the Cauchy problem for the viscoelastic wave equation for structural $\delta$-evolution models. By using the energy method in the Fourier spaces, we obtain the decay estimates of the solution to considered problem.
\end{abstract}

Key Words: Viscoelatic wave equation, Fourier transform, Lyapunov functions, Decay rates.

AMS Subject Classifications: 35L05, 35B35

\section{Introduction}

Cavalcanti et al. [3] studied the equation

$$
\left|u_{t}\right|^{\rho} u_{t t}-\Delta u-\Delta u_{t t}-\int_{0}^{t} g(t-s) \Delta u(s) d s-\gamma \Delta u_{t}=0,
$$

with $x \in \Omega, t>0, \rho>0$. They proved a global existence result for $\gamma \geq 0$, and an exponential decay for $\gamma>0$. This last result has been extended to a situation, where a source term is competing with the strong damping mechanism and the one induced by the viscosity. For more details see [9]. The authors combined well known methods with perturbation techniques to show that a solution with positive small energy exists globally and decay to the rest state exponentially.

In any spaces dimension, the paper [13] treated the viscoelastic wave equation

$$
u_{t t}-\Delta u+\int_{0}^{t} g(t-s) \Delta u(s) d s=0, \quad x \in \mathbb{R}^{n}, \quad t>0,
$$

${ }^{*}$ Corresponding author. Email addresses: a . beniani@yahoo.fr (A. Beniani), k.zennir@qu.edu.sa (Kh. Zennir), benaissa_abbes@yahoo.com (A. Benaissa) 
where $g$, being positive and nonincreasing, is the relaxation function which describes the material in consideration and $u_{0}=u(x, 0)$ and $u_{1}=u_{t}(x, 0)$ are given data. By using the energy method in the Fourier spaces, the general decay estimates of the solution is shown. In [17], the author considered the following equation

$$
\rho(x)\left(\left|u^{\prime}\right|^{q-2} u^{\prime}\right)^{\prime}-M\left(\left\|\nabla_{x} u\right\|_{2}^{2}\right) \Delta_{x} u+\int_{0}^{t} g(t-s) \Delta_{x} u(s) d s=0, \quad x \in \mathbb{R}^{n}, \quad t>0,
$$

where $q, n \geq 2$ and $M$ is a positive $C^{1}$-function satisfying for

$$
s \geq 0, \quad m_{0}>0, \quad m_{1} \geq 0, \quad \gamma \geq 1, \quad M(s)=m_{0}+m_{1} s^{\gamma} .
$$

In order to compensate the lack of Poincaré's inequality in $\mathbb{R}^{n}$ and for wider class of relaxation functions, the author used the weighted spaces to establish a very general decay rate of solutions of viscoelastic wave equations in Kirchhoff-type.

In [5], the author looked into a linear Cauchy viscoelastic equation with density. His study included the exponential and polynomial rates, where he used the spaces weighted by density to compensate for the lack of Poincaré's inequality. The same problem treated in [5], was considered in [7], where it id considered a Cauchy problem for a viscoelastic wave equation. Under suitable conditions on the initial data and the relaxation function, they prove a polynomial decay result of solutions. The used conditions on the relaxation function $g$ and its derivative $g^{\prime}$ are different from the usual conditions.

Recently, in [8], the authors considered the weak-viscoelastic case in the following problem,

$$
u^{\prime \prime}-\Delta u-\Delta u^{\prime}+\alpha(t) \int_{0}^{t} g(t-s) \Delta u(s, x) d s=0, \quad x \in \mathbb{R}^{n}, \quad t \in \mathbb{R}_{*}^{+},
$$

where $n \geq 2$. The energy decay results were established for weak-viscoelastic wave equation in $\mathbb{R}^{n}$, which depends on the behavior of both $\alpha$ and $g$. The main idea of the proof was to construct an appropriate Lyapunov function of the system obtained after taking the Fourier transform.

To extend previous results, we study the decay rate of the solution to the Cauchy problem for structural damped $\delta$-evolution with memory term in Fourier spaces

$$
u_{t t}+(-\Delta)^{\delta} u-\int_{0}^{t} g(t-s)(-\Delta)^{\delta} u(s) d s=0, \quad x \in \mathbb{R}^{n}, \quad t>0,
$$

with the initial conditions

$$
u(x, 0)=u_{0}(x), \quad u_{t}(x, 0)=u_{1}(x), \quad \text { and } \quad \delta>1 .
$$

The model here considered are well known ones and refer to materials with memory as they are termed in the wide literature which is concerned about their physical, mechanical behavior and the many interesting analytical problems. The physical characteristic property of such materials is that their behavior depends on time not only through the present time but also through their past history. 


\section{Preliminaries and assumptions and Asymptotic stability}

We assume that the function $g$ satisfies the following conditions:

A1: $g: \mathbb{R}^{+} \longrightarrow \mathbb{R}^{+}$is of class $C^{1}$ satisfying,

$$
g(0)>0, \quad 1-\int_{0}^{\infty} g(t) d t=k>0, \quad g^{\prime}(t) \leq 0, \quad \forall t \in \mathbb{R}^{+} .
$$

A2: There exists a positive nonincreasing differentiable function $\alpha(t)$ satisfying

$$
g^{\prime}(t)+\alpha(t) g(t) \leq 0, \quad \forall t \in \mathbb{R}^{+},
$$

and

$$
\alpha(t)>0, \quad \alpha^{\prime}(t) \leq 0, \quad \forall t>0 .
$$

For any real (complex)-valued function $h(t)$, we define

$$
\begin{aligned}
& (g * h)(t)=\int_{0}^{t} g(t-\tau) h(\tau) d \tau \\
& (g \circ h)(t)=\int_{0}^{t} g(t-\tau)|h(t)-h(\tau)|^{2} d \tau .
\end{aligned}
$$

For a later use, we have the following lemma, which is useful in obtaining our estimate of solutions in the Fourier space.

Lemma 2.1 ([8]). For any $f \in C^{1}\left(\mathbb{R}^{+}\right)$and any $h \in H^{1}\left(\mathbb{R}^{+}\right)$, we have

$$
\begin{aligned}
\operatorname{Re}(f * h)(t) \bar{h}_{t}(t)=- & \frac{1}{2} f(t)|h(t)|^{2}+\frac{1}{2}\left(f^{\prime} \circ h\right)(t) \\
& -\frac{1}{2} \frac{d}{d t}\left\{(f \circ h)(t)-\left(\int_{0}^{t} f(\tau) d \tau\right)|h(t)|^{2}\right\},
\end{aligned}
$$

and

$$
\left|\int_{0}^{t} f(t-s)(h(s)-h(t)) d s\right|^{2} \leq \int_{0}^{t}|f(s)| d s \int_{0}^{t}|f|(t-s)|h(t)-h(s)|^{2} d s .
$$

The following Lemma is very useful in the sequel.

Lemma 2.2 ([15]). Assume that $\beta(t)>0$ for all $t \geq 0, l, p \geq 2$. Then we have

$$
\left\||\xi|^{l} \exp \left\{-c|\xi|^{2} \int_{0}^{t} \beta(\tau) d \tau\right\}\right\|_{L^{p}} \leq C\left(1+\int_{0}^{t} \beta(\tau) d \tau\right)^{-\frac{l}{2}-\frac{N}{2 p}},
$$

where $C$ is a positive constant. 
Our aim is to obtain the decay estimates of the solution to the problem (1.5)-(1.6). After Fourier transformation of (1.5)-(1.6), we get the following system

$$
\begin{cases}\hat{u}_{t t}+|\xi|^{2 \delta}\left(\hat{u}-\int_{0}^{t} g(t-s) \hat{u}(s) d s\right)=0, & \xi \in \mathbb{R}^{n}, \quad t \geq 0, \\ \hat{u}(\xi, 0)=\hat{u}_{0}(x), \quad \hat{u}_{t}(\xi, 0)=\hat{u}_{1}(x), \quad \xi \in \mathbb{R}^{n} .\end{cases}
$$

The energy associated to (2.6) is given by

$$
\hat{E}(\xi, t)=\frac{1}{2}\left\{\left|\hat{u}_{t}\right|^{2}+|\xi|^{2 \delta}|\hat{u}|^{2}\right\}(\xi, t) .
$$

Lemma 2.3. The modified energy associated to (2.6) is defined as follows.

$$
\hat{\mathcal{E}}(\xi, t)=\frac{1}{2}\left\{\left|\hat{u}_{t}\right|^{2}+\left(1-\int_{0}^{t} g(s) d s\right)|\xi|^{2 \delta}|\hat{u}|^{2}+|\xi|^{2 \delta}(g \circ \hat{u})\right\}(\xi, t),
$$

and the modified energy $\hat{\varepsilon}(\xi, t)$ is non-increasing and satisfies for all $t \geq 0$

$$
\frac{d \hat{\varepsilon}(\xi, t)}{d t}=|\xi|^{2 \delta}\left(g^{\prime} \circ \hat{u}\right)(\xi, t)-|\xi|^{2 \delta} g(t)|\hat{u}(\xi, t)|^{2} \leq 0 .
$$

Proof. Multiplying the equation in (2.6) by $\overline{\hat{u}}_{t}$ and taking the real part, we get

$$
\frac{1}{2} \frac{d}{d t}\left\{\left|\hat{u}_{t}(\xi, t)\right|^{2}+|\xi|^{2 \delta}|\hat{u}(\xi, t)|^{2}\right\}=\operatorname{Re}\left\{|\xi|^{2 \delta} \overline{\hat{u}}_{t} \int_{0}^{t} g(s) \hat{u}(\xi, t-s) d s\right\} .
$$

Using Lemma 2.1, we easily see that

$$
\begin{aligned}
& \operatorname{Re}\left\{|\xi|^{2 \delta} \overline{\hat{u}}_{t} \int_{0}^{t} g(s) \hat{u}(\xi, t-s) d s\right\} \\
= & -\frac{1}{2}|\xi|^{2 \delta} g(t)|\hat{u}(\xi, t)|^{2}+\frac{1}{2}|\xi|^{2 \delta}\left(g^{\prime} \circ \hat{u}\right)(\xi, t) \\
& -|\xi|^{2 \delta} \frac{1}{2} \frac{d}{d t}\left\{(g \circ \hat{u})(\xi, t)-\left(\int_{0}^{t} g(\tau) d \tau\right)|\hat{u}(\xi, t)|^{2}\right\} .
\end{aligned}
$$

Substituting this last equality into (2.10), the identity (2.9) then follows.

On the other hand, using (A1) there exists a positive constant $c_{1}>0$ such that, for all $t \geq 0$ and for all $\xi \in \mathbb{R}^{n}$, we have

$$
\hat{E}(\xi, t) \leq c_{1} \hat{\mathcal{E}}(\xi, t)
$$

Thus, we complete the proof.

We will prove the following exponential stability result. 
Proposition 2.1. Let $\hat{u}(\xi, t)$ be the solution of (2.6). Then there exist two positive constants $C$, $\beta_{0}$ such that

$$
\hat{E}(\xi, t) \leq \hat{E}(\xi, 0) \exp \left\{-\frac{\beta_{0}|\xi|^{2 \delta}}{1+|\xi|^{2 \delta}} \int_{0}^{t} \alpha(s) d s\right\}, \quad \forall t \geq t_{0}>0 .
$$

To prove Proposition 2.1, the key point is to apply the multiplier techniques in Fourier spaces in order to obtain useful estimates and prepare some functionals associated with the nature of our problem to introduce an appropriate Lyapunov functional.

Proof. Multiplying (2.6) by $\overline{\hat{u}}$ and taking the real part, we get

$$
\begin{aligned}
& \frac{d}{d t} \operatorname{Re}\left(\hat{u}_{t} \overline{\hat{u}}\right)+\left(1-\int_{0}^{t} g(s) d s\right)|\xi|^{2 \delta}|\hat{u}|^{2}-\left|\hat{u}_{t}\right|^{2} \\
& \quad+|\xi|^{2 \delta} \operatorname{Re}\left\{\overline{\hat{u}}(t) \int_{0}^{t} g(t-s)(\hat{u}(t)-\hat{u}(s)) d s\right\}=0 .
\end{aligned}
$$

Applying Young's inequality, we obtain, for any $\varepsilon_{1}>0$,

$$
\begin{aligned}
& \frac{d}{d t} \operatorname{Re}\left(\hat{u}_{t} \overline{\hat{u}}\right)+\left(1-\varepsilon_{1}-\int_{0}^{t} g(s) d s\right)|\xi|^{2 \delta}|\hat{u}|^{2}-\left|\hat{u}_{t}\right|^{2} \\
\leq & \frac{1}{4 \varepsilon_{1}}|\xi|^{2 \delta}(1-k)(g \circ \hat{u})(\xi, t) .
\end{aligned}
$$

Next, the existence of the memory term forces us to make the first modification of the energy by multiplying (2.6) by $-\int_{0}^{t} g(t-s)(\overline{\hat{u}}(t)-\overline{\hat{u}}(s)) d s$ and taking the real part, we have that

$$
\begin{aligned}
& -\frac{d}{d t} \operatorname{Re}\left(\hat{u}_{t} \int_{0}^{t} g(t-s)(\overline{\hat{u}}(t)-\overline{\hat{u}}(s)) d s\right)+\operatorname{Re}\left(\hat{u}_{t} \int_{0}^{t} g^{\prime}(t-s)(\overline{\hat{u}}(t)-\overline{\hat{u}}(s t)) d s\right) \\
& \quad+\left|\hat{u}_{t}\right|^{2} \int_{0}^{t} g(s) d s-|\xi|^{2 \delta} \operatorname{Re}\left(\hat{u}(t) \int_{0}^{t} g(t-s)(\overline{\hat{u}}(t)-\overline{\hat{u}}(s)) d s\right) \\
& \quad+|\xi|^{2 \delta} \operatorname{Re}\left\{\left(\hat{u} \int_{0}^{t} g(t-s) d s\right)\left(\int_{0}^{t} g(t-s)(\overline{\hat{u}}(t)-\overline{\hat{u}}(s)) d s\right)\right\} \\
& \quad-|\xi|^{2 \delta}\left|\int_{0}^{t} g(t-s)(\hat{u}(t)-\hat{u}(s)) d s\right|^{2}=0 .
\end{aligned}
$$

Young's inequality gives, for any $\varepsilon_{2}>0$,

$$
\begin{aligned}
&- \frac{d}{d t} \operatorname{Re}\left(\hat{u}_{t} \int_{0}^{t} g(t-s)(\overline{\hat{u}}(t)-\overline{\hat{u}}(s)) d s\right)+\left|\hat{u}_{t}\right|^{2} \int_{0}^{t} g(s) d s \\
& \leq \varepsilon_{2}\left|\hat{u}_{t}\right|^{2}-\frac{g(0)}{4 \varepsilon_{2}} \int_{0}^{t} g^{\prime}(t-s)|\hat{u}(t)-\hat{u}(s)|^{2} d s+\varepsilon_{2}(2-k)|\xi|^{2 \delta}|\hat{u}|^{2} \\
& \quad+\left(|\xi|^{2 \delta}\left\{\frac{(1-k)}{4 \varepsilon_{2}}+(1-k)^{2}+\frac{1}{4 \varepsilon_{2}}\right\}\right)(g \circ \hat{u})(t), \quad \forall t \geq 0 .
\end{aligned}
$$


Then, for any $t \geq t_{0}$, the estimate (2.14) can be rewritten as follows

$$
\begin{gathered}
-\frac{d}{d t} \operatorname{Re}\left(\hat{u}_{t} \int_{0}^{t} g(t-s)(\overline{\hat{u}}(t)-\overline{\hat{u}}(s)) d s\right)+\left(\int_{0}^{t_{0}} g(s) d s-\varepsilon_{2}\right)\left|\hat{u}_{t}\right|^{2} \\
\leq \varepsilon_{2}(2-k)|\xi|^{2 \delta}|\hat{u}|^{2}-\frac{g(0)}{4 \varepsilon_{2}}\left(g^{\prime} \circ \hat{u}\right)(t)+|\xi|^{2 \delta}\left\{\frac{(2-k)}{4 \varepsilon_{2}}+(1-k)^{2}\right\}(g \circ \hat{u})(t) .
\end{gathered}
$$

Now, we define the functional $\mathcal{L}(\xi, t)$ as follows.

$$
\mathcal{L}(\xi, t)=\frac{g_{0}}{2} \operatorname{Re}\left(\hat{u}_{t} \overline{\hat{u}}\right)-\operatorname{Re}\left(\hat{u}_{t} \int_{0}^{t} g(t-s)(\overline{\hat{u}}(t)-\overline{\hat{u}}(s)) d s\right),
$$

where $g_{0}=\int_{0}^{t_{0}} g(s) d s$. Taking the derivative of $\mathcal{L}(\xi, t)$ with respect to $t$ and exploiting the estimates (2.13) and (2.15), we arrive to

$$
\begin{aligned}
& \frac{d}{d t} \mathcal{L}(\xi, t)+\frac{g_{0}}{2}\left(k-\varepsilon_{1}-\left.\varepsilon_{2}(2-k)|\xi|\right|^{2 \delta}|\hat{u}|^{2}+\left\{\frac{g_{0}}{2}-\varepsilon_{2}\right\}\left|\hat{u}_{t}\right|^{2}\right. \\
\leq & -c_{2}\left(g^{\prime} \circ \hat{u}\right)(t)+c_{3}|\xi|^{2 \delta}(g \circ \hat{u})(t), \quad \forall t \geq 0,
\end{aligned}
$$

where $c_{2}$ and $c_{3}$ are two positive constants depending on $\varepsilon_{1}$ and $\varepsilon_{2}$. In the last estimate we have used the fact that $1-\int_{0}^{t} g(s) d s \geq k$ and $g_{0}=\int_{0}^{t_{0}} g(s) d s \leq \int_{0}^{t} g(s) d s$ for any $t \geq t_{0}$.

Now, we choose $\varepsilon_{1}<k$ in (2.17) and $\varepsilon_{2}$ small enough such that $\varepsilon_{2}<\min \left(\frac{g_{0}}{2}, \frac{l-\varepsilon_{1}}{2-k}\right)$. Then there exist positive constants $\lambda_{i},(i=1,2,3)$ positive constants such that

$$
\begin{aligned}
& \frac{d}{d t} \mathcal{L}(\xi, t)+\lambda_{1}|\xi|^{2 \delta}|\hat{u}|^{2}+\lambda_{2}\left|\hat{u}_{t}\right|^{2}+\lambda_{3}|\xi|^{2 \delta}(g \circ \hat{u})(t) \\
\leq & -c_{2}\left(g^{\prime} \circ \hat{u}\right)(t)+\left(c_{3}+\lambda_{3}\right)|\xi|^{2 \delta}(g \circ \hat{u})(t), \quad \forall t \geq 0 .
\end{aligned}
$$

Define the Lyapunov functional $\mathcal{F}(\xi, t)$ as follows.

$$
\mathcal{F}(\xi, t):=\eta\left(1+|\xi|^{2 \delta}\right) \mathcal{E}(\xi, t)+|\xi|^{2 \delta} \mathcal{L}(\xi, t),
$$

for a large positive constant $\eta$ that has to be chosen later. It is straightforward to see that for $\eta$ large enough, we can find two positive constants $\beta_{1}$ and $\beta_{2}$ such that

$$
\beta_{1}\left(1+|\xi|^{2 \delta}\right) \mathcal{E}(\xi, t) \leq \mathcal{F}(\xi, t) \leq \beta_{2}\left(1+|\xi|^{2 \delta}\right) \mathcal{E}(\xi, t), \quad \forall t \geq 0 .
$$

On the other hand, taking the derivative of $\mathcal{F}(\xi, t)$ with respect to $t$ and using $(2.9)$ and (2.18), we have

$$
\begin{aligned}
& \frac{d}{d t} \mathcal{F}(\xi, t)+\lambda_{1}|\xi|^{4 \delta}|\hat{u}|^{2}+\lambda_{2}|\xi|^{2 \delta}\left|\hat{u}_{t}\right|^{2}+\lambda_{3}|\xi|^{4 \delta}(g \circ \hat{u})(t) \\
\leq & \left(\eta-c_{2}\right)|\xi|^{2 \delta}\left(g^{\prime} \circ \hat{u}\right)(t)+\left(c_{3}+\lambda_{3}\right)|\xi|^{4 \delta}(g \circ \hat{u})(t), \quad \forall t \geq 0 .
\end{aligned}
$$


At this point, we choose $\eta$ large enough such that (2.20) holds and $\eta-c_{2}>0$. Consequently (2.21) yields

$$
\frac{d}{d t} \mathcal{F}(\xi, t)+\lambda_{0}|\xi|^{2 \delta} \hat{\mathcal{E}}(\xi, t) \leq c_{4}|\xi|^{4 \delta}(g \circ \hat{u})(t), \quad \forall t \geq t_{0}
$$

for some $\lambda_{0}, c_{4}>0$.

Multiplying (2.22) by $\alpha(t)$ and making use of (2.2), we get

$$
\begin{aligned}
& \alpha(t) \frac{d}{d t} \mathcal{F}(\xi, t)+\lambda_{0} \alpha(t)|\xi|^{2 \delta} \hat{\mathcal{E}}(\xi, t) \\
\leq & c_{4} \alpha(t)|\xi|^{4 \delta}(g \circ \hat{u})(t) \\
\leq & c_{4}|\xi|^{4 \delta}\left(-g^{\prime} \circ \hat{u}\right)(t), \quad \forall t \geq t_{0} .
\end{aligned}
$$

Exploiting (2.9), we have

$$
\alpha(t) \frac{d}{d t} \mathcal{F}(\xi, t)+\lambda_{0} \alpha(t)|\xi|^{2 \delta} \hat{\mathcal{E}}(\xi, t) \leq-c_{4}|\xi|^{2 \delta} \frac{d}{d t} \hat{\varepsilon}(\xi, t), \quad \forall t \geq t_{0} .
$$

This gives

$$
\frac{d}{d t}\left\{\alpha(t) \mathcal{F}(\xi, t)+c_{4}|\xi|^{2 \delta} \hat{\mathcal{E}}(\xi, t)\right\}-\alpha^{\prime}(t) \mathcal{F}(\xi, t)+\lambda_{0} \alpha(t)|\xi|^{2 \delta} \hat{\mathcal{E}}(\xi, t) \leq 0, \quad \forall t \geq t_{0} .
$$

Recalling that $\alpha^{\prime}(t) \leq 0$ and setting

$$
\mathcal{K}(\xi, t):=\alpha(t) \mathcal{F}(\xi, t)+c_{4}|\xi|^{2 \delta} \hat{\mathcal{E}}(\xi, t),
$$

we get

$$
\frac{d}{d t} \mathcal{K}(\xi, t)+\lambda_{0} \alpha(t)|\xi|^{2 \delta} \hat{\mathcal{E}}(\xi, t) \leq 0, \quad \forall t \geq t_{0} .
$$

On the other hand, since $\alpha(t)$ is bounded, we deduce that

$$
\mathcal{K}(\xi, t) \sim\left(1+|\xi|^{2 \delta}\right) \hat{\varepsilon}(\xi, t) .
$$

These last two estimates lead to

$$
\frac{d}{d t} \mathcal{K}(\xi, t)+\beta_{0} \frac{|\xi|^{2 \delta}}{1+|\xi|^{2 \delta}} \alpha(t) \mathcal{K}(\xi, t) \leq 0, \quad \forall t \geq t_{0},
$$

for some $\beta_{0}>0$. Integrating (2.28) with respect to $t$ yields

$$
\mathcal{K}(\xi, t) \leq \mathcal{K}\left(\xi, t_{0}\right) \exp \left\{-\frac{\beta_{0}|\xi|^{2 \delta}}{1+|\xi|^{2 \delta}} \int_{t_{0}}^{t} \alpha(t) d s\right\} .
$$


Thus, using (2.27), we obtain

$$
\begin{aligned}
\hat{\mathcal{E}}(\xi, t) & \leq C \hat{\mathcal{E}}\left(\xi, t_{0}\right) \exp \left\{-\frac{\beta_{0}|\xi|^{2 \delta}}{1+|\xi|^{2 \delta}} \int_{t_{0}}^{t} \alpha(t) d s\right\} \\
& \leq C \hat{\mathcal{E}}(\xi, 0) \exp \left\{-\frac{\beta_{0}|\xi|^{2 \delta}}{1+|\xi|^{2 \delta}} \int_{0}^{t} \alpha(t) d s\right\} .
\end{aligned}
$$

Finally, the estimate (2.12) holds by combining (2.11) and (2.30). This finishes the proof of Proposition 2.1.

Thus our main result reads as follows:

Theorem 2.1. Let $\sigma$ be a nonnegative integer. Assume that $U_{0}=\left(u_{1}, u_{0}^{\prime}\right)^{T} \in H^{\sigma}\left(\mathbb{R}^{n}\right) \cap$ $L^{1}\left(\mathbb{R}^{n}\right)$. Then the solution $U=\left(u_{t}, u_{x}\right)^{T}$ of the problem (1.5) satisfies, for all $t \geq 0$, the following decay estimates

$$
\left\|\partial_{x}^{k} U(t)\right\|_{L^{2}} \leq C\left(1+\int_{0}^{t} \alpha(s) d s\right)^{-\frac{\delta k}{2}-\frac{N}{4}}\left\|U_{0}\right\|_{L^{1}}+C e^{-c \int_{0}^{t} \alpha(s) d s}\left\|\partial_{x}^{k} U_{0}\right\|_{L^{2}},
$$

where $C$ and $c$ are two positive constants and $k \leq \sigma$.

Proof. Applying the Plancherel theorem and observing that $|\widehat{U}(\xi, t)|^{2}$ and $\widehat{E}(\xi, t)$ are equivalent, and making use of (2.12), we obtain

$$
\begin{aligned}
\left\|\partial_{x}^{k} U(t)\right\|_{2}^{2}= & \int_{\mathbb{R}^{n}}|\xi|^{2 k}|\widehat{U}(\xi, t)|^{2} d \xi \\
\leq & C \int_{\mathbb{R}^{n}}|\xi|^{2 k} e^{-\frac{\beta_{0}|\xi|^{2 \delta}}{1+|\xi|^{2 \delta}} \int_{0}^{t} \alpha(s) d s}|\widehat{U}(\xi, 0)|^{2} d \xi \\
= & C \int_{|\xi| \leq 1}|\xi|^{2 k} e^{-\frac{\beta_{0}|\xi|^{2 \delta}}{1+|\xi|^{2 \delta}} \int_{0}^{t} \alpha(s) d s}|\widehat{U}(\xi, 0)|^{2} d \xi \\
& \quad+C \int_{|\xi| \geq 1}|\xi|^{2 k} e^{-\frac{\beta_{0} \mid \xi \xi^{2 \delta}}{1+|\xi|^{2 \delta}} \int_{0}^{t} \alpha(s) d s}|\widehat{U}(\xi, 0)|^{2} d \xi \\
= & : I_{1}+I_{2} .
\end{aligned}
$$

Looking carefully to the form of $\rho(\xi)=\frac{|\xi|^{2 \delta}}{1+|\xi|^{2 \delta}}$, we see that $\rho(\xi) \geq \frac{1}{2}|\xi|^{2 \delta}$ for $|\xi| \leq 1$ and $\rho(\xi) \geq \frac{1}{2}$ for $|\xi| \geq 1$.

Consequently, we infer that

$$
\begin{aligned}
I_{1} & \leq C\left\|\widehat{U}_{0}\right\|_{L_{\infty}}^{2} \int_{|\xi| \leq 1}|\xi|^{2 k} e^{-\frac{1}{2}|\xi|^{2} \delta \int_{0}^{t} \alpha(s) d s} d \xi \\
& \leq C\left(1+\int_{0}^{t} \alpha(\tau) d \tau\right)^{-k \delta-\frac{n}{2}}\left\|U_{0}\right\|_{L^{1}}^{2}
\end{aligned}
$$


where we have used Lemma 2.2

On the other hand,

$$
I_{2} \leq C e^{-\frac{1}{2} \int_{0}^{t} \alpha(s) d s} \int_{|\xi| \geq 1}|\xi|^{2 k}\left|\widehat{U}_{0}(\xi)\right|^{2} d \xi \leq C e^{-\frac{1}{2} \int_{0}^{t} \alpha(s) d s}\left\|\partial_{x}^{k} U_{0}\right\|_{L^{2}}^{2} .
$$

Collecting (2.33) and (2.34), then the estimate (2.31) holds. This completes the proof of Theorem 2.1.

\section{Acknowledgements}

The authors wish to thank deeply the anonymous referees for their useful remarks and their careful reading of the proofs presented in this paper.

\section{References}

[1] A. Beniani, A. Benaissa and Kh. Zennir, Polynomial decay of solutions to the Cauchy problem for a Petrovsky-Petrovsky system in $\mathbf{R}^{n}$, Acta. Appl. Math., 146 (2016), 67-79.

[2] S. Berrimi and S. A. Messaoudi, Exponential decay of solution to a viscoelastic equation with nonlinear localized damping, Electron. J. Diff. Eq., 88(10) (2004), 1-10.

[3] M. M. Cavalcanti, V. N. Domingos Cavalcanti and J. Ferreira, Existence and uniform decay for nonlinear viscoelastic equation with strong damping, Math. Meth. Appl. Sci., 24 (2001), 1043-1053

[4] C. M. Dafermos, Asymptotic stability in viscoelasticity, Arch. Ration. Mech. Anal., 37 (1970), 297-308.

[5] M. Kafini, Uniforme decay of solutions to Cauchy viscoelastic problems with density, Elec. J. Diff. Eq., 93 (2011), 1-9.

[6] M. Kafini and S. A. Messaoudi, On the uniform decay in viscoelastic problem in $\mathbb{R}^{n}$, Appl. Math. Comput., 215 (2009), 1161-1169.

[7] M. Kafini, S. A. Messaoudi and Nasser-eddine Tatar, Decay rate of solutions for a Cauchy viscoelastic evolution equation, Indagationes Math., 22, (2011), 103-115.

[8] M. Karek, Kh. Zennir and H. Sissaoui, Decay rate estimate of solution to damped wave equation with memory term in Fourier spaces, Glob. J. Pure and Appl. Math., 11(5) (2015), 3027-3038.

[9] S. A. Messaoudi and N. E. Tatar, Global existence asymptotic behavior for a Nonlinear Viscoelastic Problem, Math. Meth. Sci.Research J., 7(4) (2003), 136-149.

[10] S. A. Messaoudi, General decay of solution of a viscoelastic equation, J. Math. Anal. Appl., 341 (2008), 1457-1467.

[11] S. A. Messaoudi and M. I. Mustafa, On the control of solutions of viscoelastic equations with boundary feedback, Nonl. Anal. Real World Appl., 10 (2009), 3132-3140.

[12] Muhammad I. Mustafa and S. A. Messaoudi, General stability result for viscoelastic wave equations, J. Math. Phys., 53 (2012), 053702.

[13] B. Said-Houari and S. A. Messaoudi, General decay estimates for a cauchy viscoelastic wave problem, Commun. Pure Appl. Anal., 13 (2014), 1541-1551.

[14] Y. Shibata, On the rate of decay of solutions to linear viscoelastic equation, Math. Methods Appl. Sci., 23 (2000), 203-226. 
[15] J. Wirth, Asymptotic Properties of Solutions to Wave Equations with Time-Dependent Dissipation, PhD thesis, TU Bergakademie Freiberg, 2004.

[16] Xiaojun Lu and Michael Reissig, Rates of decay for structural damped models with decreasing in time coefficients, Int. J. Dynamic Systemes and Differential Equation, 2 (2009), 21-54.

[17] Kh. Zennir, General decay of solutions for damped wave equation of Kirchhoff type with density in $\mathbb{R}^{n}$, Ann Univ Ferrara, 61 (2015), 381-394.

[18] S. Zitouni and Kh. Zennir, On the existence and decay of solution for viscoelastic wave equation with nonlinear source in weighted spaces, Rend. Circ. Mat. Palermo, II. Ser, (2016), DOI 10.1007/s12215-016-0257-7. 